\title{
Design of 30-T pulsed magnetic field generator for magnetized high-energy-density plasma experiments
}

\author{
R. V. Shapovalov, ${ }^{1,2,}$ G. Brent, ${ }^{2}$ R. Moshier, ${ }^{2}$ M. Shoup, ${ }^{2}$ R. B. Spielman, ${ }^{2}$ and P.-A. Gourdain ${ }^{1,2}$ \\ ${ }^{1}$ Department of Physics and Astronomy, University of Rochester, Rochester, New York 14627, USA \\ ${ }^{2}$ Laboratory for Laser Energetics, University of Rochester, Rochester, New York 14623, USA
}

(Received 15 January 2019; published 9 August 2019)

\begin{abstract}
This paper presents the conceptual design of a high-voltage pulser intended to generate 30-T magnetic fields for magneto-inertial fusion experiments at the OMEGA facility. The pulser uses a custom capacitor bank and two externally triggered spark gaps to drive a multiturn coil. This new high-voltage pulser is capable of storing 10 times more energy than the previous system, using a higher charge voltage (from 20 to $30 \mathrm{kV}$ ) and a larger capacitance (from 1 to $5 \mu \mathrm{F}$ ). Circuit simulations show that this pulser can deliver $100 \mathrm{kA}$ into a $60-\mathrm{nH}, 14-\mathrm{m} \Omega$ coil with a rise time of $1 \mu \mathrm{s}$. For a coil with two turns with an average coil diameter of $7.8 \mathrm{~mm}$, this current translates into a 32-T peak magnetic field at coil center. This is a factor of 3 increase in the peak magnetic field compared to the present generator magnetic field capabilities.
\end{abstract}

DOI: 10.1103/PhysRevAccelBeams.22.080401

\section{INTRODUCTION}

Magnetized plasmas and magnetized, inertialconfinement-fusion (ICF) experiments are an important milestone in fusion energy research. Pioneered on the OMEGA laser [1], this research integrates basic plasma physics, transport in magnetized plasmas, alpha-particle energy deposition in ICF capsules, and magneto-inertialfusion concepts [2-6]. It also enables scientists to tackle more fundamental physics questions by studying positron focusing [7], astrophysical shocks [8-10], magnetized plasma jets, and magnetic reconnection [11]. At the OMEGA laser facility, a high-magnetic field is generated using the magneto-inertial fusion electrical discharge system (MIFEDS). The MIFEDS is a discharge pulser optimized to generate a high-current pulse into a magnetic coil. The pulser is installed inside the ten-inch-manipulator (TIM) unit and coil is positioned inside the OMEGA target chamber by moving the MIFEDS's TIM unit to the appropriate location. Unfortunately, the TIM constraints (volume and weight) fundamentally limit the energy that is available for the production of magnetic fields and the upgrade of the MIFEDS system is urgently needed.

MIFEDS-2 is a second-generation, high-voltage pulser/ coil system currently used for magnetized, high-energy plasma experiments at the OMEGA and OMEGA-EP laser

"rshapova@ur.rochester.edu

Published by the American Physical Society under the terms of the Creative Commons Attribution 4.0 International license. Further distribution of this work must maintain attribution to the author(s) and the published article's title, journal citation, and DOI. facilities [12]. The present system combines two, 500-nF (General Atomics) capacitors followed by a single spark gap (Excelitas GP-14B) switch, conical, coaxial transmission line and magnetic coil. The pulser total internal inductance is measured to be $139 \mathrm{nH}$ and the pulser internal resistance is about $130 \mathrm{~m} \Omega$. The capacitors can be charged up to $20 \mathrm{kV}$ which corresponds up to $200 \mathrm{~J}$ of initial pulser energy. Even for a low-inductance coil, these driver values limit the current to about $40 \mathrm{kA}$, making it difficult to reach a magnetic field larger than $20 \mathrm{~T}$.

In this paper, we present the design of MIFEDS-3, a new high-voltage pulser capable of generating magnetic fields greater than $30 \mathrm{~T}$. The general view of the MIFEDS-3 pulser is shown in Fig. 1. The pulser is comprised of two, custom General Atomics 2.5- $\mu \mathrm{F}$ capacitors connected in parallel and charged to a maximum voltage of $30 \mathrm{kV}$. At this charging voltage, the stored capacitor energy is $2.25 \mathrm{~kJ}$, which is 10 times larger than the energy available in the MIFEDS-2 system. This gain in energy is achieved by increasing the physical size of the capacitors, requiring the HV power supply to be moved outside of the OMEGA TIM. Each of the two pulser capacitors are each discharged through a single, triggered spark gap (Excelitas GP-14B). The estimated internal pulser inductance is $90 \mathrm{nH}$, and the internal series resistance is less than or equal to $140 \mathrm{~m} \Omega$. We believe that MIFEDS-3 gives the best magnetic-field performance possible on OMEGA given the volume and weight limitations imposed on the pulser by the TIM. MIFEDS-3, similar to MIFEDS-2, uses bubble-style packaging (the pulser is installed inside an air bubble unit, which is placed inside the TIM under vacuum) and all vacuum-air interfaces were designed with the same specifications as the MIFEDS-2 system. Because MIFEDS-3 will be charged to a voltage higher than MIFEDS-2, 30-kV 


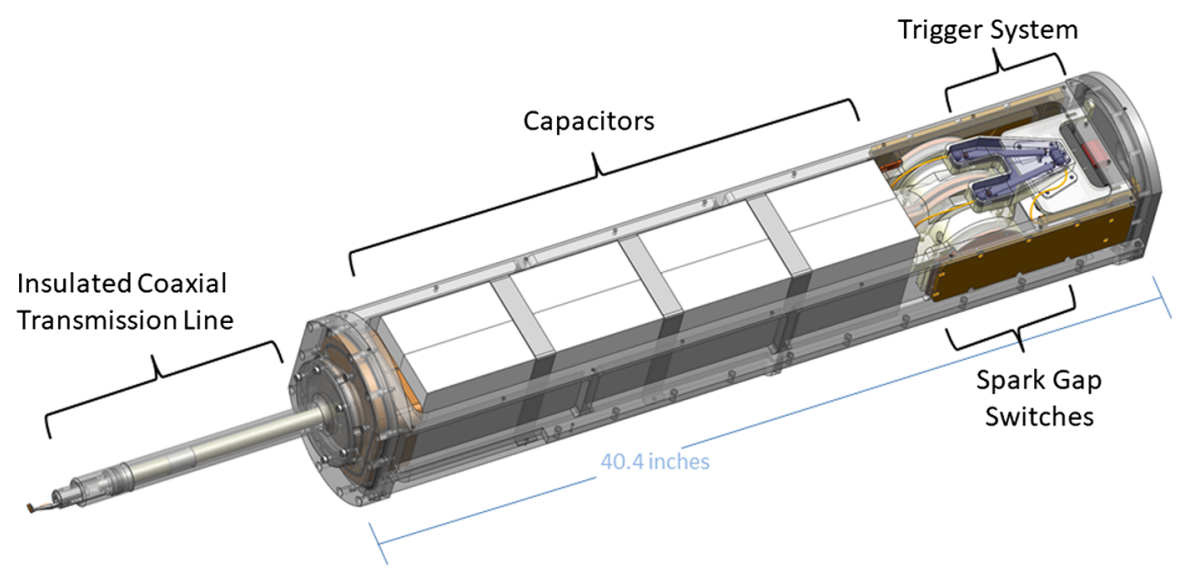

FIG. 1. MIFEDS-III design view.

voltage, to prevent any possible voltage breakdown, a plastic sleeve is inserted inside the MIFEDS-3 coaxial transmission line. We anticipate significant improvements in MIFEDS-3 performance as compared to the present MIFEDS-2 pulser.

We first present the detailed MIFEDS-3 pulser circuit simulations into a typical $60-\mathrm{nH}, 14-\mathrm{m} \Omega$ coil. We next discuss how the driver parameters scale with the coil inductance. Finally, we present the magnetic field calculations for 1-, 2-, 3-, and 4-turn coils and compare them with the MIFEDS-2 pulser. The MIFEDS-3 pulser is capable of suppling a maximum current of $100 \mathrm{kA}$. We expect that the MIFEDS-3 pulser will generate more than 30-T peak magnetic field inside the simple, 2-turn magnetic coil.

As will be shown in a following section, for lowinductance coils below $400 \mathrm{nH}$, the expected time to peak of the current is less than $2 \mu$ s and we do not expect the coil motion to significantly affect its magnetic field. Though we do not include an extended calculation of the coil's motion, heating and the physics of the skin depth on the surface of the coil, our simulations give us a solid understanding of what can be expected from the proposed MIFEDS-3 pulser and how its performance is compared to the presently in use MIFEDS-3 pulser. The comparisons of MIFEDS-3 with other compact high-magnetic field generators [13,14,15] for laser-plasma experiments is briefly discussed at the end.

\section{PULSER CIRCUIT MODELING}

We first present detailed circuit simulations into a typical MIFEDS coil. For consistency, we assume a 2-turn coil with average coil diameter of $7.8 \mathrm{~mm}$ that is made of 24-AWG (American wire gauge) copper-insulated wire. The 24-AWG wire has an outer diameter of $762 \mu \mathrm{m}$ (including insulation) and a copper core diameter of $508 \mu \mathrm{m}$. For a single turn, the coil inductance can be approximated by [16] $L \approx \mu_{0} R[\ln (8 R / r)-1.75]$, where $\mu_{0}$ is the vacuum permeability, $R$ is the coil radius, and $\mathrm{r}$ is the wire radius. The total coil resistance was simply calculated as $R=\rho l / S$, where $\rho$ is wire resistivity at room temperature, and $r$ and $S$ are the wire radius and crosssection area, correspondingly. Some discussions on why this simple formula should not affect much the driver performance will be made later. The total wire length equals to $l=N \pi d+12 \mathrm{~cm}$, where $N$ is the number of turns, $\mathrm{d}$ is the coil diameter, and $12 \mathrm{~cm}$ is an extra length of the coil wire legs. For $N=2$ turn coil, the coil inductance is $60 \mathrm{nH}$ ( $N^{2}$ times larger), the total wire length is $17 \mathrm{~cm}$, and the coil resistance is $14 \mathrm{~m} \Omega$.

\section{A. Simulations of pulser parameters into the matched 60-nH, 14-m $\Omega$ coil}

In the following simulations, we follow the "matched" load approach, $R=\sqrt{L / C}$. This is an ideal case for the high-current driver when one wants simultaneously to maximize the load current and minimize the voltage reversal (i.e., ringing) inside the system [17]. This case should not be confused with the so-called "critically matched" load case $R=2 \sqrt{L / C}$, which also is very important for pulsed-power applications, but in general delivers slightly less current as compared to the matched load case. The total pulser inductance (internal value plus coil value) is equal to $150 \mathrm{nH}$, and the total pulser resistance is matched to $\sqrt{L / C}$ and equals $170 \mathrm{~m} \Omega$. The matched value is slightly larger than the total pulser resistance (internal value plus coil value) of $154 \mathrm{~m} \Omega$ and can be compensated by installing in-series high-resistance wire, if desired. It is important to note that the MIFEDS-3 internal resistance, $140 \mathrm{~m} \Omega$ is, by design, close to the matched $\sqrt{L / C}$ pulser value, $170 \mathrm{~m} \Omega$, maximizing the load peak current and preventing the unwanted late-time current oscillations. We assume, for simplicity, no change in the wire resistance during the current pulse. This should be a good assumption, as the coil resistance is a small portion of the total pulser resistance. The total wire resistance for $N=2$ turn coil is $14 \mathrm{~m} \Omega$ which accounts for only $10 \%$ compared to the MIFEDS-3 internal inductance. In this case, the increase in the coil resistance due to the coil 


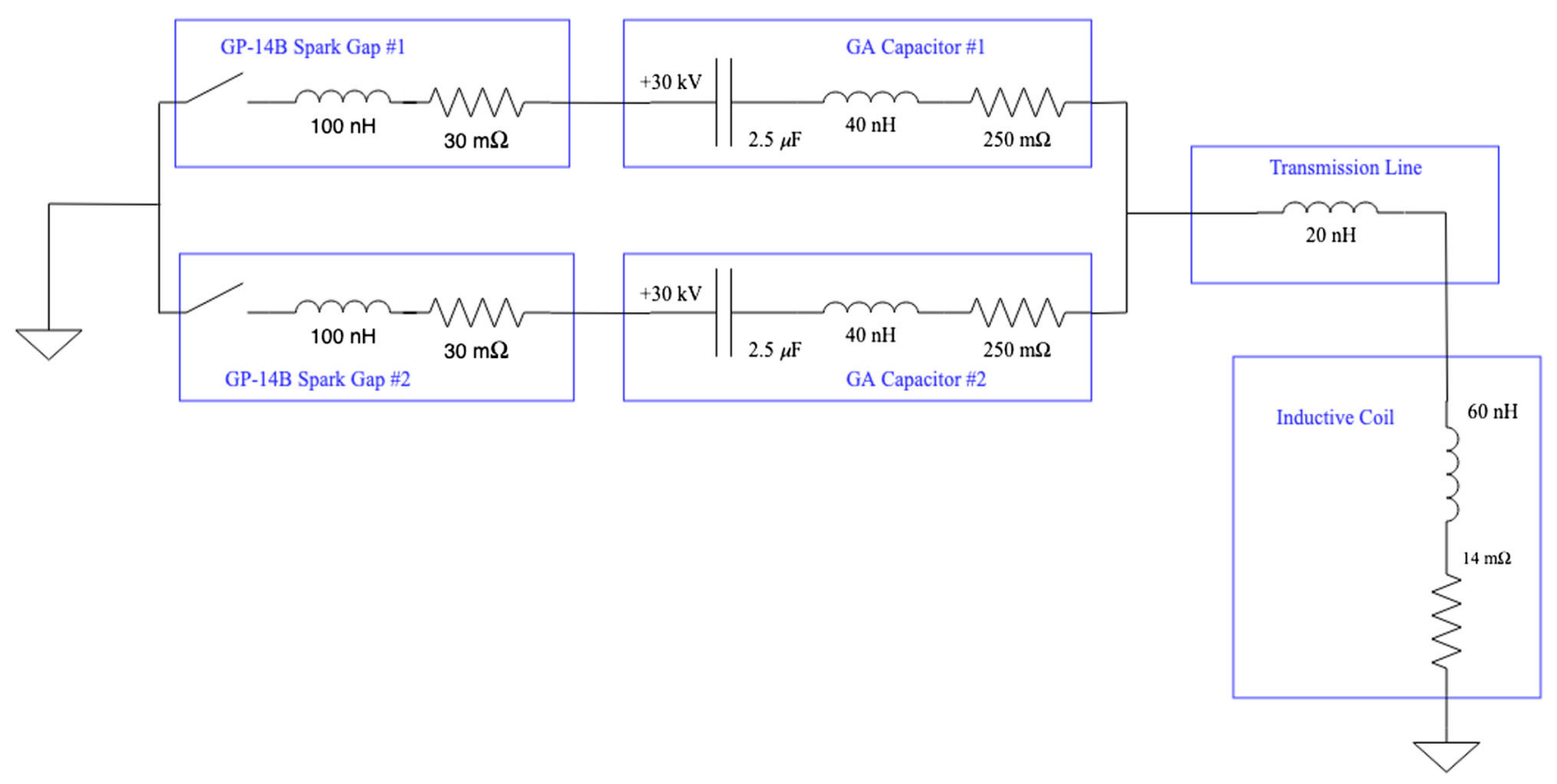

FIG. 2. MIFEDS-III circuit diagram.

heating and plasma development should not affect much the total driver performance. All simulations are performed with the SCREAMER $[18,19]$, a special-purpose pulsedpower circuit simulations tool.

The MIFEDS-3 pulser circuit diagram is shown in Fig. 2. Each of the two, 2.5- $\mu$ F GA capacitors is charged to $30 \mathrm{kV}$ and each is triggered through a single, triggered spark gap GP-14B. The inductance of each capacitor is about $40 \mathrm{nH}$ and its equivalent series resistance is about $30 \mathrm{~m} \Omega$. The inductance of the switch is about $100 \mathrm{nH}$ and its resistance is about $250 \mathrm{nH}$. The inductance of the transmission line is estimated to be around $20 \mathrm{nH}$ which bridged with $60-\mathrm{nH}$, 14-m $\Omega$ 2-turn coil described above. Though, the total pulser internal inductance can be estimated to be equal to $(100 \mathrm{nH}+40 \mathrm{nH}) / 2+20 \mathrm{nH}=90 \mathrm{nH}$, and the total internal resistance is about $(30 \mathrm{~m} \Omega+250 \mathrm{~m} \Omega) / 2=140 \mathrm{~m} \Omega$.

The simulations of the current and voltage waveforms are presented in Fig. 3. A peak current of 95 kA is reached $1.05 \mu \mathrm{s}$ after the start of the current waveform (the quartercycle time). The maximum coil voltage is $12 \mathrm{kV}$ at the beginning of the coil pulse, and the minimum voltage is $-2.8 \mathrm{kV}$ at about $2.4 \mu \mathrm{s}$ after the pulse starts. The resistive part of the voltage drop, thin dashed line, reaches its maximum value of $1.9 \mathrm{kV}$ at $1.05 \mu \mathrm{s}$, and the inductive part of the voltage, thin dot-dashed line, follows closely the total voltage applied to the coil. We observe that both the positive and the negative peak currents are well inside the capacitor's safety margins. The negative reversal current is $-16 \mathrm{kA}$ at a time of $\sim 4 \mu$ s with minimal current oscillations afterward. The presented pulser design, being very close to the matched load circuit, optimizes the coil peak current but limits the pulser current oscillations at later times.
It is interesting to compare the stored electrical energy of the pulser with the electrical energy delivered to the coil at the time of peak current, $1.05 \mu \mathrm{s}$. The total energy stored in the pulser is $2.25 \mathrm{~kJ}$. The total energy delivered to a 60-nH coil, including its resistive and inductive parts, is plotted in Fig. 4. The peak energy delivered to the coil is $\sim 400 \mathrm{~J}$ or $\sim 18 \%$ of the initial energy stored in the capacitor. The resistive energy heats the coil and by the time of the current peak only $42 \%(\sim 110 \mathrm{~J} / \sim 260 \mathrm{~J})$ of the total resistive contribution is deposited in the coil. The inductive part of the energy, as expected, reaches its maximum value of about $270 \mathrm{~J}$ at the time of the peak current. The MIFEDS-3 pulser delivers $12 \%$ of the initially

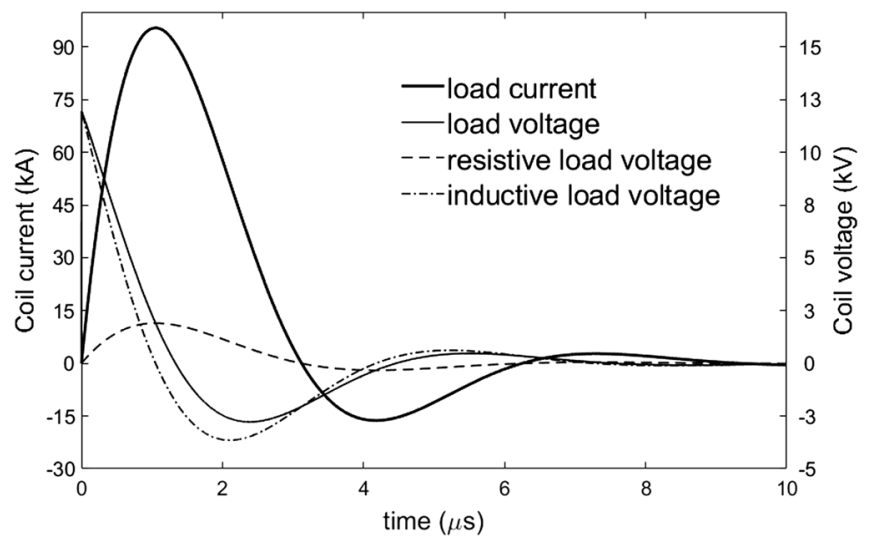

FIG. 3. Simulations of the current and voltage waveforms into a matched $60-\mathrm{nH}$ coil are shown. The thick solid line is the total load current, the thin solid line is the load total load voltage, the dashed line is the load resistive voltage, and the dot-dashed line is the load inductive voltage. 


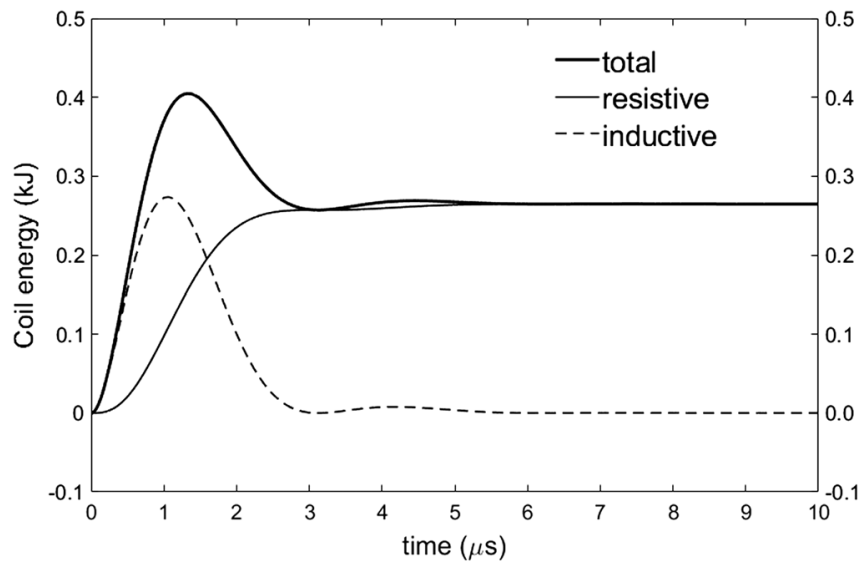

FIG. 4. The energy deposition calculations into a matched 60$\mathrm{nH}$ coil. The thick solid line is the total energy delivered to the coil, the thin solid line is its resistive part, and the thin dotted line is its inductive part.

stored electrical energy into the magnetic energy of this 60 -nH coil. It is tempting to increase the time to peak of the current (larger capacitors) to increase the total energy stored inside the magnetic field, but longer current rise times will result in increased motion (expansion) of the coil wires, preventing the coil from reaching its maximum value of peak magnetic field. Keeping this current rise below 1-2 $\mu$ s gives an optimal coupling between the initial stored energy and the magnetic energy. Current rise times shorter than $1-\mu \mathrm{s}$ result in excessive coil voltages when the peak current is increased beyond MIFEDS-2 currents.

\section{B. Scaling of the pulser's parameters with the coil inductance}

Other experiments may require different coils and we are interested in the dependence of the pulser output parameters on coil inductance. The most important pulser parameters affecting the resulting coil magnetic field are the pulser peak current and the time-to-peak current. A scan of these pulser parameters as a function of coil inductance is presented in Fig. 5. Each data point is a separate SCREAMER run with the coil inductance value from the horizontal axis. The total driver inductance is, as before, the sum of the pulser internal inductance plus the inductance of the corresponding coil. However, we do not assume the matched, $\sqrt{L / C}$, resistance value as we did earlier for 2-turn, 60-nH coil. Instead, we assume the constant coil resistance of $20 \mathrm{~m} \Omega$, and the constant total pulser resistance of $160 \mathrm{~m} \Omega(140 \mathrm{~m} \Omega+20 \mathrm{~m} \Omega)$ for all SCREAMER runs for all pulser coils. A more accurate estimate of the coil resistance will be possible when the coil wire length is better known.

As expected, the pulser peak currents and time-to-peak currents are monotonic functions of the coil inductance. As we increase the coil inductance from $100 \mathrm{pH}$ to $100 \mathrm{nH}$, the peak currents decrease from $115 \mathrm{kA}$ down to $50 \mathrm{kA}$,

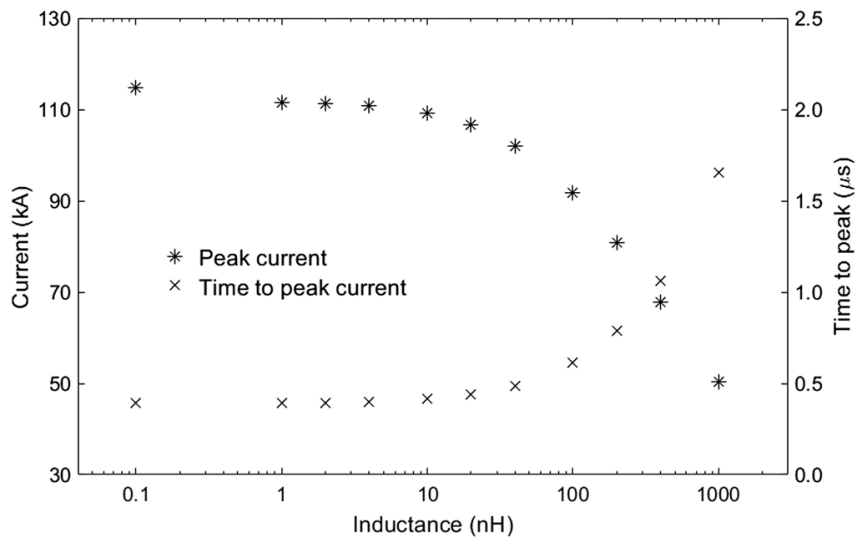

FIG. 5. MIFEDS-3 coil peak current (asterisk) and time-to-peak current (times) calculations as a function of coil inductance. The coil resistance is assumed to be $20 \mathrm{~m} \Omega$ for all cases.

and time-to-peak currents increase from 800 ns up to $3.3 \mu \mathrm{s}$. For low-inductance coils below $40 \mathrm{nH}$, the peak currents are slightly above the operational pulser limit of $100 \mathrm{kA}$ (switch and capacitor limited). For the highinductance coils above $400 \mathrm{nH}$, the time-to-peak currents become larger than $2 \mu \mathrm{s}$, and the coil performance will degrade because of the increased wire motion. These calculations suggest that the optimal pulser performance is achieved when the coil inductance is in the range of 40 to $400 \mathrm{nH}$.

\section{CALCULATION OF COIL MAGNETIC FIELD}

Once the coil geometry is established and the coil current is simulated, the coil magnetic field can be calculated. This task is not trivial and usually requires numerical simulations. However, for the simple coil geometries, and if the coil shape is preserved on the time scale of the peak current, the coil magnetic field can be approximated with a simple formula. That is a good assumption for coils with inductance below $400 \mathrm{nH}$, because, as was already discussed, the time-to-peak current for such coils is below $2 \mu \mathrm{s}$, and the coil movement can be neglected. In the case of $N$-turn coil, the peak magnetic field at the coil center can be approximated by $B_{0}=\mu_{0} N I / d$, where $I$ is the coil current, $N$ is the number of the coil turns, and $d$ is the coil diameter. In this case, the magnetic field waveform, $B(t)$, is simply the scaled function of the current waveform, $I(t)$, which can be readily calculated with SCREAMER, or any other circuit code.

\section{A. Estimations of the magnetic field into a simple, 2-turn, $60-\mathrm{nH}$ coil}

Here we aim to calculate the magnetic field generated inside a typical coil and compare the coil performance with the present, MIFEDS-2, pulser. The most direct approach to compare MIFEDS-2 and MIFEDS-3 pulsers is without the matched load, $\sqrt{L / C}$, assumption. In this case, the 

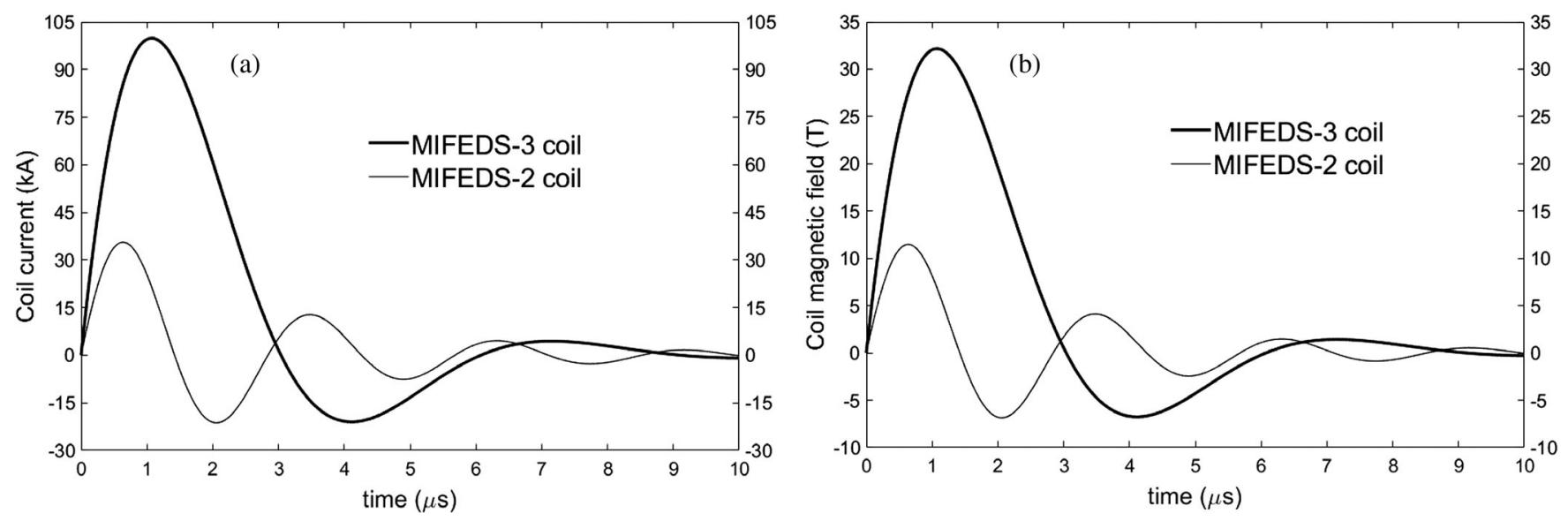

FIG. 6. Simulations of the currents and magnetic field waveforms into the 60-nH, 14-m $\Omega$ coil. Part (a) corresponds to the MIFEDS coil current and (b) shows the peak magnetic field in the coil.

pulser total resistance is a sum of its internal value $(130 \mathrm{~m} \Omega$ for MIFEDS-2, and $140 \mathrm{~m} \Omega$ for MIFEDS-3) and the resistance of the coil. Once again, we consider a simple, 2-turn coil with average coil diameter of $7.8 \mathrm{~mm}$ made of 24-AWG copper insulated wire. The inductance and resistance of this coil was calculated to be $60-\mathrm{nH}$ and $140 \mathrm{~m} \Omega$, respectively. The rest of the pulser parameters are as discussed in the Introduction.

Both the current and magnetic field calculations into a 2-turn, 7.8-mm diameter coil are presented, Fig. 6. As can be seen, for the new MIFEDS-3 pulser, the simulations predict maximum 100-kA peak current at about $1.1 \mu \mathrm{s}$. Not a surprise, this is a factor of 3 larger than MIFEDS-2 current. It is interesting to observe that the MIFEDS-3 current waveform is very close to the matched load case presented in Fig. 3. The slight difference in the peak and time-to-peak values accounts for the driver resistance mismatch as compared to the matched load. The coil magnetic fields [Fig. 6(b)] are simply the scaled waveforms of the coil currents. The peak magnetic field generated inside the MIFEDS-3 coil reaches $32 \mathrm{~T}$ by the time of the current peak $(1.1 \mu \mathrm{s})$, which is, as expected, the factor of 3 larger as compared to the present MIFEDS-2 pulser.

The FWHM for MIFEDS-3 current and magnetic field waveforms is about twice as large as the MIFEDS-2 driver. In magnetized, high-energy-density experiments on OMEGA, the lasers are triggered at the current peak [12], a time interval where the current change is limited to $\pm 5 \%$ (also called the flattop window). The flattop window is about $770 \mathrm{~ns}$, relaxing the allowable jitter in triggering MIFEDS-3, compared to MIFEDS 2, which has a 380-ns window.

\section{B. Dependence of the coil magnetic field on the total number of turns}

In experiments calling for a stronger magnetic field, it is possible to increase the total number of turns in the coil, keeping the coil inductance the same by reducing the coil diameter. It is also possible to increase the coil magnetic field keeping the coil diameter the same but increasing the total number of turns. Both cases are equally possible and result in the larger coil magnetic field. However, in the first case, the resulting magnetic field is concentrated over a small coil volume, and in the former case, the magnetic field is applied to the larger coil volume.

Here, we consider the generation of higher magnetic fields using four different coils, all having the same coil diameter, but with 1,2,3, and 4 total number of turns. To be consistent with our previous simulations, we assume all coils made of the same 24-AWG copper-insulated wire with $7.8-\mathrm{mm}$ average coil diameter. The inductance and resistance of these coils are calculated utilizing the same approach used for the 2-turn coil. The coil inductances are $15,60,135$, and $240 \mathrm{nH}$ with resistances of $12,14,16$, and $18 \mathrm{~m} \Omega$, for coils having 1,2,3, and 4 turns, respectively. Using SCREAMER, we simulate the current $I(t)$ waveform, and, later, using the formula for magnetic field $B_{0}=$ $\mu_{0} N I / d$, calculate the resulting coil magnetic field, $B(t)$. The current waveform simulations for coils with a different number of turns are presented in Fig. 7(a) and the corresponding magnetic field waveforms are plotted in Fig. 7(b).

It is not obvious that an increase in the total number of turns, $N$, will result in a larger magnetic field. Indeed, as we increase the total number of turns, the coil inductance increases, and the coil peak current, $I(t)$, decreases. Because the magnetic field, $B(t)$, is proportional both to $I(t)$ and $N$, the resulting magnetic field is not obvious. As can be seen from Fig. 7, left plot, the peak current gradually decreases as we increase the coil inductance. There is as much as 110-kA peak current with a 1-turn, 15-nH coil. The current decreases to about $80 \mathrm{kA}$ for 4-turn, 240-nH coil. The peak magnetic fields monotonically increase as we increase the total number of turns. The peak magnetic fields are 18, 32, 42, and $50 \mathrm{~T}$ for the coils having 1, 2, 3, and 4 turns, correspondingly. It appears that the coil current, $I(t)$, is a weaker function than the total number 

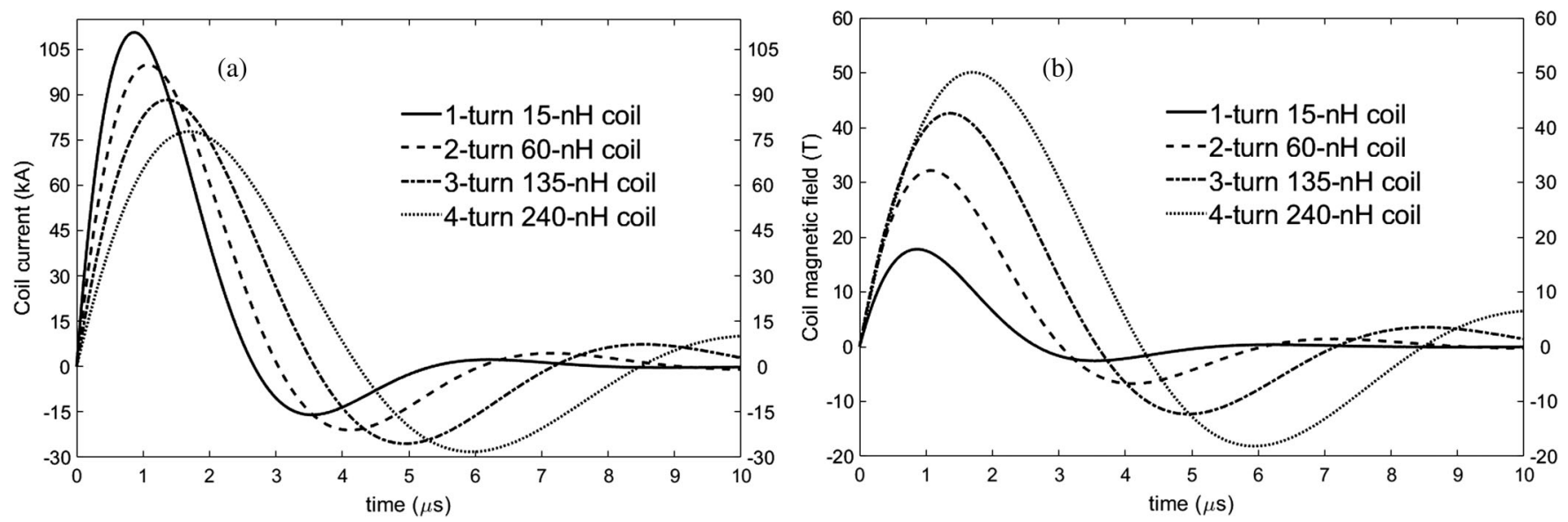

FIG. 7. MIFEDS-3 simulations into 1-, 2-, 3- and 4-turn coils. All coils have the same 7.8-mm average coil diameter. The left plot shows the SCREAMER current waveform simulations, and the right plot shows the corresponding coil magnetic field waveforms.

of turns, $N$, and the resulting magnetic field becomes larger as we increase the coil number of turns.

It should be also noted that, as we increase the number of turns, the time-to-peak current increases as well. It appears that 1-4-turns are the optimal number of coil windings for the case with 7.8-mm average coil diameter. A larger number of turns will increase the time-to-peak current above $2 \mu \mathrm{s}$, which will result in motion of the coil wires during the pulse and a degradation of the peak magnetic field.

\section{Comparison of MIFEDS-3 with MIFEDS-2 pulser and other compact high-magnetic field generators}

The comparison of the calculated peak magnetic fields for the proposed new MIFEDS-3 pulser and presently in operation MIFEDS-2 system is presented in Fig. 8. We assume 1-turn, 2-turn, 3-turn, and 4-turn coils having inductances of $15,60,135$, and $240 \mathrm{nH}$, respectively, both for MIFEDS-3 and MIFEDS-2 pulsers calculated as

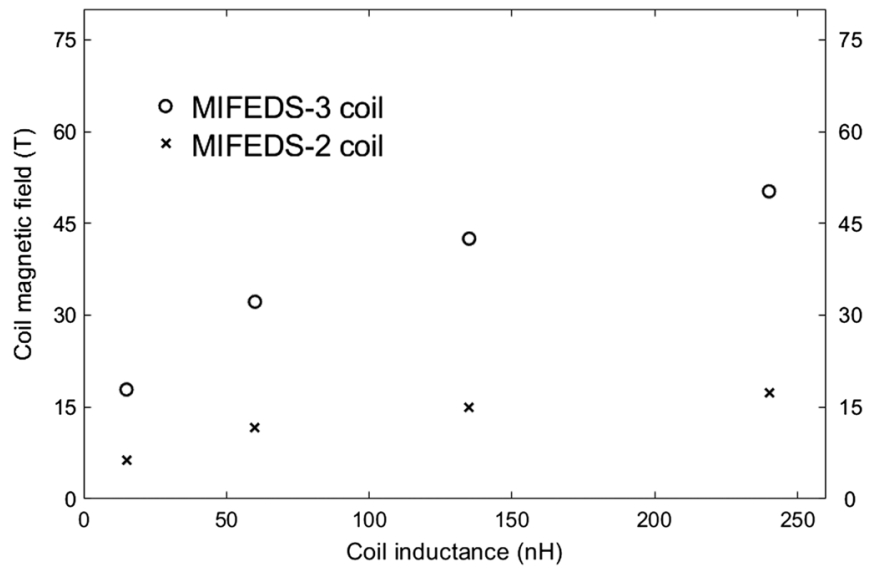

FIG. 8. Calculations of peak magnetic fields are plotted as a function of coil inductance. All coils have the same average coil diameter of $7.8 \mathrm{~mm}$. The coil inductances are 15, 60, 135, and $240 \mathrm{nH}$ for 1-turn, 2-turn, 3-turn, and 4-turn coils, respectively. described in the previous section. The peak magnetic fields for the new MIFEDS-3 pulser are from Fig. 7, right plot. The pulser internal circuit parameters, both for MIFEDS-2 and MIFEDS-3 systems, were discussed throughout the text and, for clarity, are summarized here. The internal inductance, resistance, and total capacitance are $1 \mu \mathrm{F}, 139 \mathrm{nH}$, and $130 \mathrm{~m} \Omega$ for MIFEDS-2 pulser, and $5 \mu \mathrm{F}, 90 \mathrm{nH}$, and $140 \mathrm{~m} \Omega$ for MIFEDS-3 pulser, correspondingly. The MIFEDS-2 was initially charged $20 \mathrm{kV}$, and MIFEDS-3 to $30 \mathrm{kV}$. As can be seen, the peak magnetic fields generated with the new pulser are the factor of 3 larger than peak magnetic fields generated inside the present MIFEDS-2 system. The increase in the peak magnetic fields is directly related to the capabilities of the upgraded pulser to supply the larger currents into the predefined magnetic coil. Also, the saturation of the peak magnetic fields can be seen as a function of the coil inductances-coil number of turns. For this coil with 7.8-mm diameter it is not practical to go to a higher magnetic field by increasing the number of coil's turn above 4 . In addition to the saturation effect, the time-to-peak current exceeds $2 \mu \mathrm{s}$ and the peak magnetic fields can degrade because of the increased coil motion.

The results presented in this paper can be scaled for different coil diameters and, in this way, the driver performance can be compared with some other compact high-magnetic field generators for laser-plasma experiments. The coil magnetic field is, both, proportional to the coil current, and inversely proportional to the coil diameter, as given by $B_{0}=\mu_{0} N I / d$. But the coil current is only a weak function of the coil diameter, and, in the first order, the coil magnetic field can be scaled as the inverse of the coil diameter. For the single-turn coil, the scaling relation can be written as $B_{1} / B_{2} \sim d_{2} / d_{1}$. For example, our simulations predict that $7.8-\mathrm{mm}$ diameter single-turn coil can generate 18-T peak magnetic field at the coil center (Fig. 7 or Fig. 8). Using the scaling relation just discussed above, we can estimate that about $14 \mathrm{~T}$ peak magnetic field is expected for 1-cm diameter, single-turn coil assuming no 
change in the peak current. That is close to $13.9 \mathrm{~T}$ peak magnetic field when more careful analysis, taking into the account the current simulations, is performed.

Finally, we briefly discuss how the MIFEDS-3 performance is compared to some other high-magnetic field generators for laser plasma experiments [13-15], such as, two-capacitor and ten-capacitor pulsers currently in operation at the Texas Petawatt and the Z-Beamlet laser facilities. Both pulsers are portable, have a relatively small footprint, and can be moved back and forth [13] between Texas Petawatt and Z-Beamlet for the magnetized laser plasma experiments. The Sandia two-capacitor highmagnetic field pulser [15] is comprised of two capacitorswitch blocks each connected by six coaxial cables to the triplate transmission line leading to the magnetic coil inside the vacuum chamber. It has a total capacitance of $6.2-\mu \mathrm{F}$ and system inductance of about $150 \mathrm{nH}$, which are very close to the MIFEDS-3 parameters (5- $\mu \mathrm{F}$ capacitance and 90-nH internal inductance). However, the Sandia twocapacitor system can be charged to larger $100 \mathrm{kV}$ voltage as compared to maximum $30 \mathrm{kV}$ for MIFEDS-3 pulsers. This higher charging voltage drives the higher peak current, which, in turn, results in the higher magnetic field coil's performance. When charged to the maximum $100 \mathrm{kV}$ voltage, the two-capacitor pulser can deliver as much as $500-\mathrm{kA}$ peak current with about $1.5-\mu$ s time to peak, which is factor of 5 larger than what is expected from the MIFEDS-3 pulser. According to $B_{0}=\mu_{0} N I / d$, for $1-\mathrm{cm}$ diameter, single-turn coil, this peak current translates into 60-T peak magnetic field assuming no coil motion and other effects degrade the coil magnetic field performance.

The ten-capacitor pulser [15] is a larger counterpart of the two-capacitors system with similar design but ten capacitor-switch blocks arranged around the vacuum chamber instead of two. The pulser is intended to deliver 2-MA peak current and generate up to $200 \mathrm{~T}$ peak axial magnetic field into the single-turn, low-inductance coil. The testing reveals just above 1-MA peak current with 1.7- $\mu$ s time to peak into single-turn, 5-nH coil when the pulser was half charged to $50 \mathrm{kV}$. The peak magnetic field for this coil was estimated to be over $100 \mathrm{~T}$.

Of course, the higher magnetic field capabilities of the Sandia two-capacitor and ten-capacitors systems (60 T and $200 \mathrm{~T}$ versus $14 \mathrm{~T}$ for MIFEDS-3 pulser for 1-cm singleturn coil) are a direct consequence of the fact that the Sandia pulser has higher initial stored energy, which results in a higher peak current and a larger peak magnetic field. Unfortunately, the volume available inside the TIM unit, where the MIFEDS-3 pulser is intended to be installed, does not give to us the capability to farther increase the stored energy. We believe that MIFEDS-3 pulser design presented in this paper gives the best magnetic-field performance possible on the OMEGA laser facility given the volume and the weight limitations imposed on the pulser by the TIM. We would need to move the capacitors outside of the TIM units to increase the available stored energy inside the MIFEDS-next-generation pulser to farther increase the peak magnetic field of the coil.

\section{CONCLUSION}

We describe the electrical design of the new MIFEDS-3 pulser. By increasing the current capabilities by a factor of 3 , the proposed system can achieve stronger magnetic fields, while staying within the weight and volume constraints of the TIM. The MIFEDS-3 design has a total stored electrical energy 10 times larger than its predecessor and should reach 100-kA peak current in less than $1 \mu \mathrm{s}$, into a $60-\mathrm{nH}, 14-\mathrm{m} \Omega$ coil. Assuming a 2-turn coil geometry with an average coil diameter of $7.8 \mathrm{~mm}$ this peak current gives a peak magnetic field of $32 \mathrm{~T}$ at the center of the coil. Even larger magnetic fields can be achieved using coils with more turns.

\section{ACKNOWLEDGMENTS}

This material is based upon work supported by the Department of Energy National Nuclear Security Administration under Award No. DE-NA0001944, the University of Rochester, and the New York State Energy Research and Development Authority. This report was prepared as an account of work sponsored by an agency of the U.S. Government. Neither the U.S. Government nor any agency thereof, nor any of their employees, makes any warranty, express or implied, or assumes any legal liability or responsibility for the accuracy, completeness, or usefulness of any information, apparatus, product, or process disclosed, or represents that its use would not infringe privately owned rights. Reference herein to any specific commercial product, process, or service by trade name, trademark, manufacturer, or otherwise does not necessarily constitute or imply its endorsement, recommendation, or favoring by the U.S. Government or any agency thereof. The views and opinions of authors expressed herein do not necessarily state or reflect those of the U.S. Government or any agency thereof.

[1] T. R. Boehly, D. L. Brown, R. S. Craxton, R. L. Keck, J. P. Knauer, J. H. Kelly, T. J. Kessler, S. A. Kumpan, S. J. Loucks, S. A. Letzring, F. J. Marshall, R. L. McCrory, S. F. B. Morse, W. SekaJ. M. Soures, and C. P. Verdon, Initial performance results of the OMEGA laser system, Opt. Commun. 133, 495 (1997).

[2] O. V. Gotchev, P. Y. Chang, J. P. Knauer, D. D. Meyerhofer, O. Polomarov, J. Frenje, C. K. Li, M. J.-E. Manuel, R. D. Petrasso, J. R. Rygg, F. H. Séguin, and R. Betti, LaserDriven Magnetic-Flux Compression in High-EnergyDensity Plasmas, Phys. Rev. Lett. 103, 215004 (2009).

[3] J. P. Knauer, O. V. Gotchev, P. Y. Chang, D. D. Meyerhofer, O. Polomarov, R. Betti, J. A. Frenje, C. K. Li, M. J.-E. Manuel, R. D. Petrasso, J. R. Rygg, and F. H. Séguin, 
Compressing magnetic fields with high-energy lasers, Phys. Plasmas 17, 056318 (2010).

[4] P. Y. Chang, G. Fiksel, M. Hohenberger, J. P. Knauer, R. Betti, F. J. Marshall, D. D. Meyerhofer, F. H. Seguin, and R. D. Petrasso, Fusion Yield Enhancement in Magnetized Laser-Driven Implosions, Phys. Rev. Lett. 107, 035006 (2011).

[5] M. Hohenberger, P. Y. Chang, G. Fiksel, J. P. Knauer, R. Betti, F. J. Marshall, D. D. Meyerhofer, F. H. Seguin, and R. D. Petrasso, Inertial confinement fusion implosions with imposed magnetic field compression using the OMEGA Laser, Phys. Plasmas 19, 056306 (2012).

[6] D. H. Barnak, J. R. Davies, R. Betti, M. J. Bonino, E. M. Campbell, V. Yu. Glebov, D. R. Harding, J. P. Knauer, S. P. Regan, A. B. Sefkow, A. J. Harvey-Thompson, K. J. Peterson, D. B. Sinars, S. A. Slutz, M. R. Weis, and P.-Y. Chang, Laser-driven magnetized liner inertial fusion on OMEGA, Phys. Plasmas 24, 056310 (2017).

[7] H. Chen, G. Fiksel, D. Barnak, P. Y. Chang, R. F. Heeter, A. Link, and D. D. Meyerhofer, Magnetic collimation of relativistic positrons and electrons from high intensity laser-matter interactions, Phys. Plasmas 21, 040703 (2014).

[8] A. S. Liao, S. Li, P. Hartigan, P. Graham, G. Fiksel, A. Frank, J. Foster, and C. Kuranz, Numerical simulation of an experimental analogue of a planetary magnetosphere, High Energy Density Phys. 17, 38 (2015).

[9] D. B. Schaeffer, W. Fox, D. Haberberger, G. Fiksel, A. Bhattacharjee, D. H. Barnak, S.X. Hu, and K. Germaschewski, Generation and Evolution of HighMach-Number Laser-Driven Magnetized Collisionless Shocks in the Laboratory, Phys. Rev. Lett. 119, 025001 (2017).

[10] D. B. Schaeffer, W. Fox, D. Haberberger, G. Fiksel, A. Bhattacharjee, D. H. Barnak, S. X. Hu, K. Germaschewski, and R. K. Follett, High-Mach number, laser-driven magnetized collisionless shocks, Phys. Plasmas 24, 122702 (2017).

[11] G. Fiksel, W. Fox, A. Bhattacharjee, D. H. Barnak, P. Y. Chang, K. Germaschewski, S. X. Hu, and P. M. Nilson,
Magnetic Reconnection between Colliding Magnetized Laser-Produced Plasma Plumes, Phys. Rev. Lett. 113, 105003 (2014).

[12] G. Fiksel, A. Agliata, D. Barnak, G. Brent, P.-Y. Chang, L. Folnsbee, G. Gates, D. Hasset, D. Lonobile, J. Magoon, D. Mastrosimone, M. J. Shoup III, and R. Betti. Note: Experimental platform for magnetized high-energy-density plasma studies at the OMEGA laser facility, Rev. Sci. Instrum. 86, 016105 (2015).

[13] N. J. Riley, S. M. Lewis, M. L. Wisher, M. W. Kimmel, K. W. Struve, J. L. Porter, R. D. Bengtson, and T. Ditmire, Improved experimental resolution of the Vishniac overstability in scaled late-stage supernova remnants, High Energy Density Phys. 22, 64 (2017).

[14] K. W. Struve, J. L. Porter, and D. C. Rovang, Megagauss Field Generation for High-Energy-Density Plasma Science Experiments, Sandia Report No. SAND2008-7015, 2008.

[15] K. W. Struve, J. W. Argo, R. D. Bengtson, D. I. Headley, J. W. Kellogg, S. M. Lewis, J. L. Porter, H. J. Quevedo, M. E. Savage, B. Stoltzfus, C. J. Waugh, and M. Wisher, LDRD Final Report on Confinement of Cluster Fusion Plasmas with Magnetic Fields, Sandia Report No. SAND2011-8500, 2011.

[16] F. W. Grover, Inductance Calculations (Dover Publications, Mineola, 2004).

[17] M. G. Mazarakis and R. B. Spielman, A compact, highvoltage E-beam pulser, in Proceedings of the 12th IEEE International Pulsed Power Conference, Monterey, CA (IEEE, Piscataway, NJ, 1999), https://doi.org/10.1109/ PPC.1999.825498.

[18] M. L. Kiefer and M. M. Widner, Screamer-a singleline pulsed-power design tool, in 5th IEEE Pulsed Power Conference, Washington, DC (IEEE, Piscataway, NJ, 1985).

[19] R. B. Spielman and Y. Gryazin, Screamer v4.0-a powerful circuit analysis code, in 2015 IEEE Pulsed Power Conference, Austin, TX (IEEE, Piscataway, NJ, 2015), https://doi.org/10.1109/PPC.2015.7296979. 